\title{
Practice Changing Results in RCC come from ASCO 2021
}

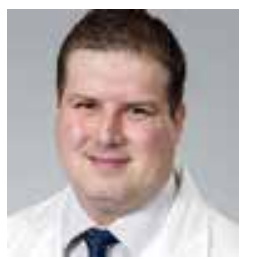

Marc R. Matrana, MD, MS, FACP

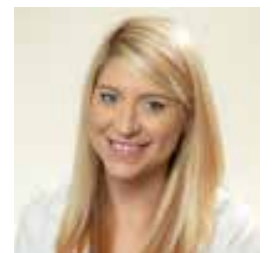

Erin Pierce, MSN, APRN, FNP-C

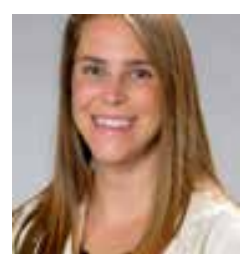

Erica Doubleday, MSN, APRN, FNP-C

Ochsner Precision Cancer Therapies Program and Ochsner Cancer Institute, Benson Cancer Center. New Orleans LA

https://doi.org/10.52733/KCJ19n2-s1-a12

$\mathrm{F}$ or the second year in a row, the annual meeting of the American Society of Clinical Oncology (ASCO) was held virtually due to the ongoing COVID-19 pandemic. Nonetheless, the meeting was hailed as a great success and brought much practice changing data in the field of genitourinary medical oncology, including kidney cancer.

\section{Adjuvant Therapy in RCC}

The most ground-breaking presentation was given by Dr. Toni Choueiri, who presented interim results of Keynote-564,aphase3 double-blindtrial comparing adjuvant pembrolizumab to placebo following nephrectomy in clear cell RCC patients with intermediateand high-risk of recurrence. The study enrolled 994 patients with pT2 grade 4 or sarcomatoid, pT3-4 any grade, node positive disease (any grade, any $\mathrm{T}$ stage) RCC within 12 weeks of nephrectomy and those with completely resected oligometastatic disease within a year of nephrectomy. The great majority of patients fell into the first two categories. The results represented the first prespecified analysis after approximately 265 disease free survival (DFS) events. DFS at 12 months was 76.2 in the control arm vs $85.7 \%$ in pembrolizumab arm and at 24 months was $68.1 \mathrm{vs} 77.3 \%$ in those arms. This represented a hazard ratio (HR) of
0.68 (95\% CI: $0.53-0.87, \mathrm{p}=0.0010$ ). Overall survival (OS) was not mature and only included about a quarter of events, but still trended towards a meaningful improvement with a $46 \%$ reduction in the risk of death in those receiving pembrolizumab, $\mathrm{HR}=0.54$ (95\% CI 0.30 - 0.96, $\mathrm{p}=0.0164)$. Safety was as expected with no new signals emerging. This represents the first positive adjuvant immunotherapy trial in RCC and in the opinion of many in the genitourinary medical oncology community, the first step towards a viable adjuvant option in RCC, given the overall weak efficacy and unfavorable toxicity profile of sunitinib in this setting. Yet, there is still more work to be done. As noted above, the OS was not mature and many questions remain, including role of PD-L1 status as a biomarker of adjuvant benefit of immunotherapy, how non-clear cell might respond to this approach, and the benefit patients with early stage disease or who are more than 12 weeks from nephrotomy might benefit, among others. Fortunately, several ongoing studies are investing some of these issues and the Keynote- 564 data continues to mature.

\section{Novel Therapies and Combina- tions}

Yuan-Yuan $\mathrm{Qu}$ and colleagues from China provided impressive updated results from a phase II study of the antiPD-L1 camrelizumab plus famitinib, a TKI against VEGFR-2, PDGFR, c-kit, and FGFR, in patients with advanced, heavily pre-treated RCC and urothelial carcinoma. Thirty-eight patients with advanced RCC were included, with an ORR of $63.2 \%$ (95\% CI, 46.078.2; 24 PRs), DCR of 89.5\% (95\% CI, 75.9-95.8), and median DOR which was not reached (range 2-19+ mos) in this cohort. Median PFS was also not reached, and 12-mo OS rate was $88.0 \%$. 92.1\% of subjects had reduction in target lesions with a median reduction of $47 \%$ from baseline noted. DCR was $100 \%$ (95\% CI, 77.2-100.0) in untreated RCC patients and 84.0\% (95\% CI, 65.393.6) in pre-treated patients. Further study of this regimen certainly seems warranted.

Results of a phase $1 \mathrm{~b}$ study of the novel IL-2v immunocytokine Simlukafusp a were presented by Perez-Garcia et al. This study enrolled 69 patients with unresectable clear cell and/or sarcomatoid RCC who were treated with the novel drug paired with atezolizumab or bevacizumab or both. The triplet combination was found to be most efficacious with ORR of 47$48 \%$ including 3 complete responses in patients treated with triplet therapy.

Dr. Nizar Tannir of MD Anderson presented results of the highly anticipated CANTATA study, which randomized 444 patients who 
had progressed on 1-2 prior lines of therapy to a combination of the glutaminase inhibitor telaglenastat plus cabozantinib or placebo plus cabozantinib. Unfortunately, the study did not meet its endpoints. Median PFS (mPFS) was 9.2 months for telaglenastat plus cabozantinib arm vs 9.3 months for the control arm (HR $=0.94 ; 95 \%$ CI: $0.74,1.21$; stratified log-rank $\mathrm{p}=$ 0.65) with overall response rates (ORR; confirmed) of $31 \%$ with Tela+Cabo vs $28 \%$ Pbo+Cabo, respectively.

Tumor growth in Von HippelLindau disease is driven by an aberrant accumulation of HIF-2alpha due to the inactivation of $\mathrm{VHL}$, ultimately resulting in the development of clear cell RCC, pancreatic neuroendocrine tumors (pNET), and hemangioblastomas. A phase 2 study of the HIF-2alpha inhibitor belzutifan (MK-6482) in patients with Von Hippel-Lindau disease associated RCC revealed promising results. 61 patients were enrolled with a median follow-up of 69 weeks, of those, 92\% remained on therapy at the time of analysis. There were 22 confirmed responses (36\%) and (11\%) unconfirmed responses. ORR was $80 \%$ in pNETs including one complete response and $32 \%$ in CNS hemangioblastomas,

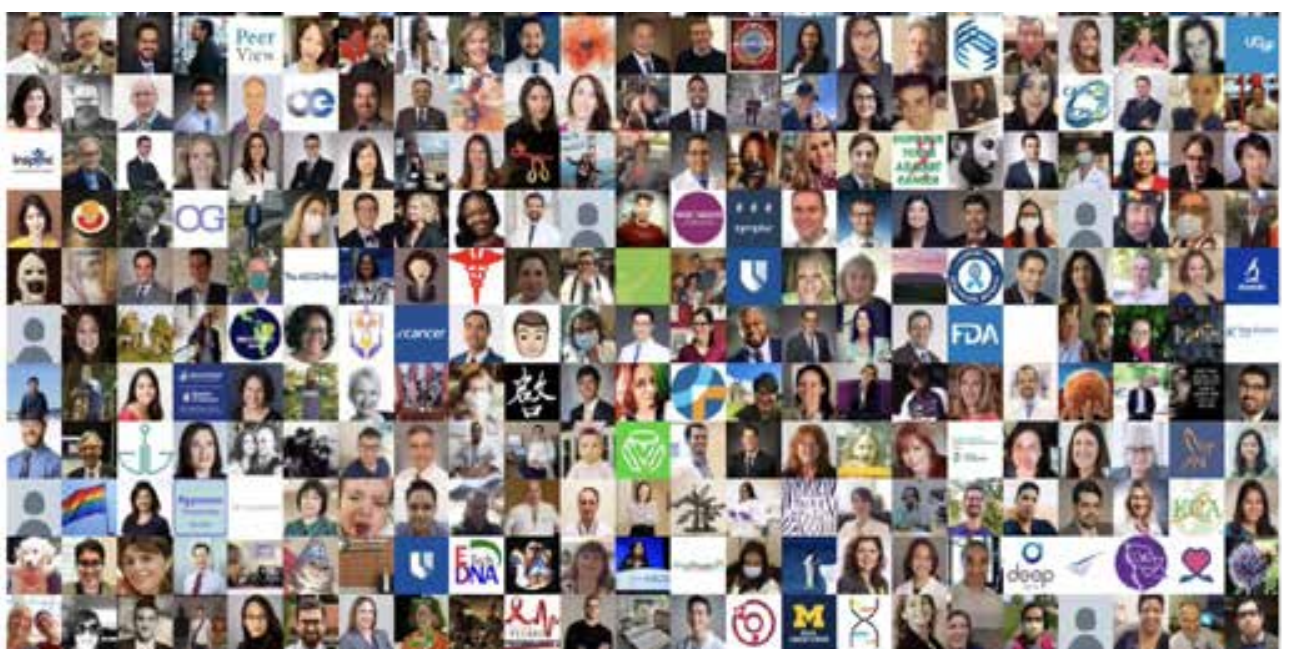

nivolumab plus ipilimumab alone (11\%). Median PFS was also prolonged (NR vs 11 weeks), although sample size was small (30 subjects) and the comparator arm did much poorer than historically expected.

Xinan Sheng and colleagues from China presented a Phase 3 double-blind three arm trial in which randomized patients received in a 1:1:1 fashion either the VEGF-targeted vorolanib plus placebo, everolimus, or a combination of these.

399 patients were enrolled. Median PFS was prolonged in combination arm compared to the single-agent everolimus group (10.0 months [95\% CI, 8.2-10.4] vs. 6.4 months [95\% CI, 4.7-8.3]; HR = 0.70 [95\% CI, 0.52-0.94]; $\mathrm{P}=0.0171) . \quad$ Median PFS was similar between single-agent vorolanib and single-agent everolimus (6.4 months [95\% CI, 4.6-8.3] vs. 6.4 months [95\% CI, 4.7-8.3]; HR = 0.94 [95\% CI, 0.69-
RCC the first line setting were presented. Previous analysis showed the combination significantly improved OS, PFS, and ORR vs sunitinib monotherapy in treatment-naive advanced clear cell $\mathrm{RCC}$ and the combination gained FDA approval. Overall, 861 subjects were randomized with 418 deaths at this 42-month analysis. The combination of pembrolizumab plus axitinib improved OS compared to sunitinib (median: 45.7 vs $40.1 \mathrm{mo}$; HR, 0.73 [95\% CI: $0.60-$ o.88]; $\mathrm{P}<0.001$ ) and PFS (median: 15.7 vs $11.1 \mathrm{mo}$; HR, 0.68 [95\% CI, 0.58$0.80]$; $\mathrm{P}<0.0001)$. The $42-\mathrm{mo}$ OS rate was $57.5 \%$ with combination vs $48.5 \%$ in the control arm; the 42-mo PFS rate was $25.1 \%$ with the combination vs $10.6 \%$ with sunitinib. ORR for the combination was $60.4 \%$ vs $39.6 \%$ with suntinib $(\mathrm{P}<0.0001)$, and a CR rate of $10.0 \%$ vs $3.5 \%$ in the combination arm and control arm, respectively. No new safety signals were observed in this long-term analysis. also including one complete response. There were 16 retinal hemangioblastoma patients evaluable at baseline, of which $69 \%$ showed improvement.

There is much research around the role of the microbiome in cancer, and particularly around response to immunotherapy. Results of a randomized phase $1 \mathrm{~b}$ study of CBM588 (an agent principally consisting of Clostridium butyricum) showed a significantly improved ORR when this drug was added nivolumab plus ipilimumab (59\%) as compared to
1.24]; $P=0.6856)$. Response was achieved by $33 / 133$ (24.8\%) of patients in the combination arm, $11 / 133$ (8.3\%) in the single-agent everolimus arm, and $14 / 133(10.5 \%)$ of those receiving singleagent vorolanib. OS was immature at the time of the analysis. The combination was associated with Grade 3 or greater treated related AEs in $72 \%$ of subjects.

\section{Trial Updates}

Final results of the phase 3 KEYNOTE-426 study of pembrolizimab plus axitinib vs sunitinib in metastatic with nivolumab from the TITAN-RCC study. In this tailored immunotherapy approach, patients who did not respond initially were given between 2-4 doses of nivolumab + ipilimumab as boost cycles and patients who had PR or CR initially on nivolumab monotherapy were eligible for nivolumab + ipilimumab at progression. Primary endpoint was objective response rate in first line and second line whereas the secondary endpoints included activity in nivolumab monotherapy, remission rate of patients who receive the nivolumab + ipilimumab boost and 
safety and overall survival. Results showed that the TITAN tailored immunotherapy approach improved response rates in patients compared to those patients who had received only nivolumab monotherapy with nearly half of patients receiving nivolumab + ipilimumab boosts with PD improving to PR/CR (18\%) or SD (30\%).

Health-related quality-of-life analysis from the phase 3 CLEAR study was presented by Robert Motzer. The trial, which showed that lenvatinib plus pembrolizumab improved PFS, OS, and ORR compared to sunitinib, also found the combination of lenvatinib plus pembrolizumab was associated with similar better symptoms and quality of life than sunitinib. Improvements in physical functioning, fatigue, dyspena, and constipation were significantly improved with the combination when compared with sunitinib.

Updated data on the durability of response and overall survival from the TIVO-3 study of tivozanib versus sorafenib were provided. The study enrolled patients with metastatic RCC who failed 2 or 3 prior systemic therapies, one of which included a VEGFR-targeted TKI. There were 41 responders (23\%) in the tivozanib and 20 responders $(11 \%)$ in the sorafenib. The median duration of response was 20.3 months (95\% CI: 9.8 - 29.9) with tivozanib and and 9.0 months (95\% CI: 3.7 -16.6) with sorafenib. The HR for overall survival favored tivozanib at 0.91 (95\% CI: 0.716 - 1.165).

An interesting study of outcomes of second-linetherapy patients who progressed on the JAVELIN Renal 101 study was presented by Laurence Albiges. She and her colleagues analyzed outcomes of 163 patients who received single agent therapy (including 60 who received cabozantinib) and 41 patients who received combination therapy (most commonly levantinib and everolimus) following avelumab plus axitinib on study. 36 months OS was $44.1 \%$ in those receiving single-agent therapy (95\% CI: $35.9-52.0$ ) and 63.4 months (95\% CI: $45.7-76.6$ ) in those receiving combination therapy in the second-line setting. Median secondline PFS was 20.4 months (95\% CI: 17.6 - 23.0) and 24.1 months (95\% CI: 17.7 $\mathrm{NE}$ ) in these two groups, respectively.

\section{Comparisons of First Line Therapies}

For many physicians, the question of which first-line combination therapy to use in patients with newly diagnosed metastatic RCC is daunting given the number of new combinations approved. This is exacerbated by a lack of head-tohead comparison studies among these combinations. Two ASCO abstracts attempted to compare regimens outside of the scope of a prospective head-tohead trial.

Kevin Zarrabi and colleagues abstracted data from $821 \mathrm{mRCC}$ patients from Flatiron databases, 259 who received axitinib plus pembrolizumab and 562 who received ipilimumab plus nivolumab. Demographics and clinical parameters were well matched between the two cohorts. Median age was 66 years, $73 \%$ were male, and $54.9 \%$ had a nephrectomy. The adjusted median OS between the two treatment groups was not statistically different. Twelvemonth survival was $68.5 \%$ for those receiving axitinib plus pembrolizumab and $65.8 \%$ for received ipilimumab plus nivolumab treated patients $(\mathrm{P}=0.41)$. Twelve-month real world PFS was $41.4 \%$ for the axitinib plus pembrolizumab group and $39.7 \%$ for those in the received ipilimumab plus nivolumab group $(\mathrm{P}=0.14)$.

Bradley Alexander McGregor and colleagues take a slightly different approach using a matching-adjusted indirect comparison (MDIC) of data from the Checkmate 9ER and Keynote-426 studies of nivolumab plus cabozantinib and pembrolizumab plus axitinib respectively. Nivolumab plus cabozantinib was associated with a median PFS of 19.3 compared to 15.7 months with pembrolizumab plus axitinib, and nivolumab plus cabozantinib also achieved superior overall response rate (ORR) and duration of response (DoR). OS outcomes were similar.

\section{Cytoreducitve Nephrectomy}

The role of cytoredutive nephrectomy in RCC in the targeted and immunotherapy era continues to be debated.

An abstract by Jaleh Fallah and colleagues explored nephrectomy prior to immunotherapy based combinations via a pooled analysis of data from 5 trials. Among 849 patients with Stage IV RCC at initial diagnosis, $62 \%$ had nephrectomy prior to immunotherapy based combinations. Median OS was not reached in the prior nephrectomy group, but appeared prolonged in those having surgery prior to systemic immunotherapy, even when adjusted for age and prognostic risk scores (HR=0.59, 95\% CI: 0.46-0.75).

In a similar retrospective study, Pooja Ghatalia et al extracted data on 1,719 mRCC patients from the Flatiron Health database, and found that $972 \quad(56.5 \%)$ received systemic treatment alone, $605(35.1 \%)$ received cytoreductive nephrectomy followed by systemic treatment, and 142 (8.2\%) received systemic followed by cytoreductive nephrectomy. Cytoreductive nephrectomy prior to systemic therapy was significantly associated with prolonged OS compared to systemic therapy alone, $\mathrm{HR}=0.82$, (95\% CI: 0.70-0.95, p=0.008). Taken together, these two retrospective studies suggest that cytoreductive nephrectomy may play a role in improving outcomes in certain patients and that still more studies of this controversial area are needed, especially as the landscape of system therapy continues to evolve.

\section{Non-Clear Cell RCC}

Several abstracts reported data on therapies for non-clear cell RCC. Due to the rarity of metastatic collecting duct carcinoma, the BONSAI trial was opened as a phase 2 evaluating the primary endpoint of objective response rate of patients taking cabozantinib 6omg orally once daily. From January 2018 
to November 2020, 25 patients were enrolled and 23 were treated. Six patients had stable disease, 1 with a CR, and 7 with a PR. Secondary endpoints showed an ORR of $35 \%$ and median PFS of 6 months. Treatment showed optimistic efficacy and acceptable patient tolerability in this patient population.

Gopalakrishnan et al. conducted a retrospective analysis of 203 patients with renal cell carcinoma with any sarcomatoid component and had received systemic therapy from 6 US academic cancer centers evaluating the overall survival and response rates. It was found that median overall survival and response rates were significantly higher in patients who had previously received immune checkpoint inhibitors compared to those patients who had not. For instance, median overall survival was $31 \%$ with immune checkpoint inhibitor versus $7.6 \%$ and a $66.2 \%$ disease control rate with immunotherapy versus $39.1 \%$ nonimmunotherapy drugs. Additionally, there was a large benefit noted among patients who had non clear cell and mixed histology sarcomatoid renal cell carcinoma.

In the Oracle study,
investigators wanted insight to determine if there was antitumor activity with new combination therapies such as IO, VEGF, and mTOR inhibitors in patients with metastatic nCCRCC. Research shows that despite this patient population having inferior rates with new novel combination therapies than clear cell RCC, there is some antitumor activity observed which warrants further prospective studies.

Rodriguez et al. evaluated the combination of savolitinib 1500mg every 4 weeks and durvalumab 600mg every day in MET-driven, metastatic papillary renal cell carcinoma in a single arm phase I/II trial. Investigators analyzed the confirmed response rate, PFS, tolerability and overall survival of the 41 patients who received treatment. The confirmed response rate in MET driven patients was $57 \%$ with a duration of response of 9.4 months, and a median PFS of 10.5 months. OS was 27.4 months (95\% CI: 7.3-NR). Clinical activity of this combination seems promising for MET driven papillary renal cell carcinoma patients.

The results of a phase 2 trial of patients with advanced nccRCC on Cabozantinib and nivolumab were presented by Lee et al. Patients with no history of prior immune checkpoint inhibitors and up to 1 prior line of therapy with measurable disease received cabozanitinib 40mg plus nivolumab 240mg every 2 week versus 480 mg every 4 weeks in two different cohorts. Cohort 1 consisted of 40 patients with papillary, unclassified, or translocation associated RCC and cohort 2 consisted of 7 patients with chromophobe histology. Overall results showed that there is promising efficacy in metastatic non-clear cell RCC pts with papillary, unclassified, or translocation associated histologies. ORR for the papillary, unclassified, or translocation cohort was 48\% (95\% CI 31.5-63.9; Table), and a median PFS of 12.5 months (95\% CI 6.3-16.4) and median OS was 28 months (95\% CI 16.3-NE). Unfortunately, none of the 7 chromophobe patients has a response suggesting there is limited efficacy in this sub-population.

In part 2 of UNISON (ANZUP 1602), investigators evaluated the results of treating patients with rare variant RCC refractory to single-agent nivolumab with salvage ipilimumab and nivolumab. 41 patients were determined to be refractory to single agent nivolumab and had variant of nccRCC histologies. One complete response and 3 partial responses were noted. Disease control rate at 6 months was $45 \%$ with a median PFS of 2.6 months. The primary endpoint of this study was not met. The results from this study show that although a small portion of nccRCC refractory to nivolumab may benefit from combination therapy with ipilimumab and nivolumab, the majority do not, and more research is needed to identify valuable treatment options for nccRCC patients.

\section{Potential Biomarkers}

The search for biomarkers in RCC continues. Soleimani et al evaluated plasma exosome microRNAs (miRNAs) as a potential biomarker of response to immune checkpoint-based therapy. Prior to initiating immunotherapy, $11 \mathrm{miR}$ NAs that are over-expressed in RCC and/or immune-associated were evaluated in 40 patients and in 30 healthy volunteers. RT-PCT was used to evaluate MiRNA expression between the two groups and compared using the $2 \mathrm{DDCt}$ method. The most common first line immunotherapy was nivolumab + ipilimumab, followed by pembrolizumab + axitinib, and avelumab + axitinib. Results showed that a lower expression of miR-155 was associated with response to immunotherapy in patients with metastatic RCC and highlight the need for additional research in this area for use of miR-155 as a biomarker of response.

Previously, the 27-gene immuno-oncology signature has been shown to be associated with value to immune checkpoint inhibitors in breast, lung, and bladder cancers. Investigators aimed to identify if this could also be applied to patients with renal cell carcinoma. Patients who had a positive score by the 27-gene signature had significantly better one-year PFS compared to patients with a negative score (hazard ratio $=0.235,95 \% \mathrm{CI}=$ $0.069-0.803, p<0.01$ ). Given that four tumor types validated this algorithm, additional research is needed to support this assay as a pan-cancer immune system classifier.

Tucker et al presented a retrospective review of patients seeing to evaluate and correlate baseline neutrophil-to-eosinophil ratio (NER) and neutrophil-to-lymphocyte ratio (NLR) with treatment outcomes with ipilimumab and nivolumab in patients with metastatic RCC. 111 patients were included in the analysis and it was found that patient with NER less than median had prolonged median PFS, OS, ORR as compared to those with NER greater than the median. Data suggested that 
baseline NER may serve as an early response predictor for immunotherapy with ipilimumab and nivolumab.

\section{Sites of Metastases}

Previous studies have described RCC with metastatic disease to the pancreas as a distinct clinical entity with a more indolent course as compared to historical controls. Cassandra Duarte and colleagues presented an analysis of 229 patients from 9 institutions. They report a median OS for all patients from time of metastatic disease was prolonged at 7.7 years (95\% CI 6.3-10.3). The median OS for those who received a first line VEGF targeted therapy was 7.6 years (95\% CI 5.5-9.5) and was the median OS was not reached for those receiving first-line immunotherapy (95\%CI 6.5-NR). The difference was significant with an unadjusted p-value of 0.029 , with a pair-wise comparison between median OS of first line immunotherapy subgroup compared to that of the first line VEGF inhibitor group also achieving statistical significance $(p=0.0148)$. These results suggest the choice of first-line therapy in mRCC with pancreatic metastases may impact outcome.

Patients with brain metastases are often excluded from clinical trials, limiting data on outcomes in this populations. CheckMate 920 is an ongoing phase $3 \mathrm{~b} / 4$ trial of nivolumab and ipilimumab in patients with advanced RCC with unmet medical need, including those with asymptomatic brain metastases. Of the $25 \mathrm{mRCC}$ patients with brain metastases who were evaluable for response, the ORR was $32.0 \%$ ( $95 \% \mathrm{CI}, 14.9-53.5$ ). The median duration of response was 24.0 months with $50 \%$ of responders without reported progression.

\section{Oligoprogressive Disease}

Raquibul Hannan et al presented data from a prospective phase II single arm trialofmRCCpatientswhodemonstrated response to systemic therapy and then had subsequent radiographic evidence of three or fewer sites of disease progression were treated. These patients underwent stereotatic ablative radiation (SAbR) to all progressive sites. The study found that SAbR extended PFS by $>6$ months in $70.6 \%$ of the 17 evaluable oligoprogressive mRCC patients. SAbR was safe and did not adversely affect QOL, suggesting it is a reasonable alterative to switching systemic therapies in patients with limited progression in a small number of metastatic sites.

\section{Healthcare Disparities}

Janvi Wadiwala and colleagues highlight access issues with regards to palliative care among RCC patients. In a National Cancer Database study of 50,405 patients found that socioeconomic barriers such as age, race, Spanish/Hispanic origin, income, education, and other factors affected metastatic renal cell carcinoma patients access to palliative care resources. Older patients, African Americans, higher income, and lower education status patients were less likely to receive palliative care. High education attainment and treatment at an integrated cancer center or comprehensive community cancer center increased the odds of receiving palliative care.

Gender differences in health outcomes represent another important area of healthcare disparity research, Claud Grigg and colleagues explored survival trends between men and women with metastatic RCC. Clear cell renal cell carcinoma is twice as common in men as in women, and previous studies have shown improved outcomes in women with localized RCC as compared to men. In this retrospective NCDB study, patients were grouped by date of metastatic diagnosis into three eras, pre-Tyrosine Kinase Inhibitor (TKI), TKi and immune checkpoint inhibitors (ICI). Demographic features were similar, with females being slightly older and more likely to be black. Women were found to be at an increased risk of death in both the ICI era and the
TKI era, with a worse prognosis than men that could not be explained by demographic differences.

\section{Patient Voice}

Results from a global survey of 2,012 patients and caregivers from 41 countries were reported by Rachel $\mathrm{H}$. Giles and colleagues. They found more than half (52\%) of the responders lacked understanding of subtype of diagnosis, but also reported that a similar number (51\%) felt they were as involved as they wanted to be with their care. Between 41-45\% reported the no one discussed clinical trials with them and the no one explained their likelihood of surviving their cancer beyond 5 years. These results help guide provider attention to areas where patient comprehension and needs can be better addressed.

\section{COVID-19 in RCC}

Of course, last but certainly not least is the impact that the COVID-19 pandemic has had on RCC patients. A retrospective study by Jesus Garcia Donas et al compared patients with advanced RCC who developed COVID while undergoing antiangiogenic treatment vs immunotherapy vs matched controls. Patients with RCC who developed COVID19 had treatment held more frequently and presented with lower clinical benefit rates than those not infected. Those patients on immunotherapy required more frequent interruptions and longer hospitalizations than those on antiangiogenics.

As the world continues to address the global pandemic, cancer has not slowed down. Thankfully, the fight against cancer also hasn't slowed down either as evidenced by the remarkable progress exemplified in the ASCO 2021 Annual Meeting. It is our hope that we can all join together once again in Chicago for ASCO 2022. 